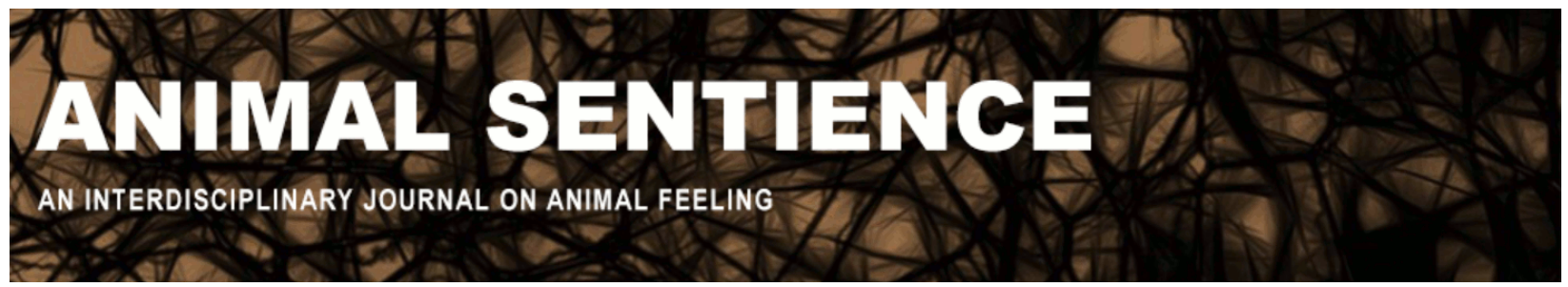

Rogers, Lesley J. (2017) Considering side biases in vigilance and fear. Animal Sentience 15(4)

DOI: $10.51291 / 2377-7478.1274$

Date of submission: 2017-12-11

Date of acceptance: 2017-12-14 (c) 


\title{
Considering side biases in vigilance and fear
}

\author{
Commentary on Beauchamp on Fear \& Vigilance
}

\author{
Lesley J. Rogers \\ School of Science and Technology \\ University of New England, NSW, Australia
}

\begin{abstract}
Measures of vigilance and fear might be more consistently associated if side biases are taken into account, because the right side of the brain is specialised to detect predators and to express fear responses. In species with eyes positioned laterally and with relatively small binocular fields, this brain asymmetry is manifested as eye preferences because each eye sends most of its input to be processed in the opposite side of the brain. Hence, responses elicited by stimuli on the animal's left side are more likely be associated with fear than are responses to the same stimuli on the animal's right side.
\end{abstract}

Lesley J. Rogers is Professor Emeritus at the University of New England, Australia, and a Fellow of the Australian Academy of Science. She has studied animal behaviour for many years and is well-known for her research on development and lateralized behaviour in the chicken. She has also published on welfare in domestic chickens and other species.

https://www.une.edu.au/staff-profiles/science-and-technology/Irogers

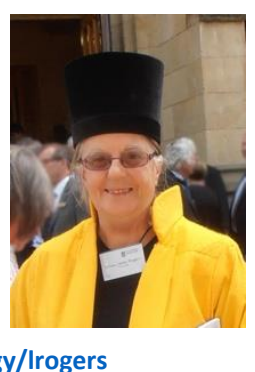

In the target article, Beauchamp (2017) explains convincingly that vigilance is not always associated with fear and hence cannot always be relied on as a putative indicator of the level of fear. I agree entirely and here I would like to offer a potential way of measuring vigilance that may have a more reliable association with fear.

This might be achieved by taking into account asymmetry of the brain regions involved in the detection of threats and in expressing fear. As shown in a number of species (fish, reptiles, amphibians, birds and primates), the right side of the brain (right hemisphere) is specialized to respond to predators and novel stimuli and to express fear responses (summarised in MacNeilage et al., 2009, and Rogers et al., 2013). One of the brain regions with a role in fear behaviour is the amygdala, and the right amygdala shows greater neuronal changes than the left amygdala in response to fear conditioning (Scicli et al., 2004; Young and Williams, 2013). In humans also, the right amygdala has a specialized role in fear responses (Morris et al., 1999). This means that stronger fear responses are expressed to potentially dangerous stimuli detected in the left monocular visual field, as seen clearly in animals with laterally positioned eyes, because each eye has a large monocular visual field which sends inputs to the opposite side of the brain.

Austin and Rogers (2007) showed that domestic horses exhibit stronger avoidance (fear) responses when approached on their left side by a human opening an umbrella (a looming stimulus) compared to the same approach on their right side. Feral horses show a similar left-side bias in vigilance and reactivity (Austin and Rogers, 2012) and so too do undomesticated Przewalski 
horses (Austin and Rogers, 2014). Toads, and also marsupial mice, are more reactive to an approaching model snake seen in their left monocular visual field (Lippolis et al., 2002,2005) and chicks are more likely to respond to a model avian predator when they detect it in the left visual field (Rogers, 2000). Although other markers of fear (e.g., heart rate) have yet to be measured in these test conditions, the laterality of the response suggests that the association between vigilance and fear might be stronger when the eliciting stimulus is on the animal's left side than when it is on the right side. Hence, taking side bias into account may refine the measured link between vigilance and fear.

This specialisation of the left eye and right hemisphere to respond to predators has been demonstrated clearly in field studies of Australian magpies. On hearing their species-specific "eagle" call, magpies tilt their head to scan overhead using their left eye (Rogers and Kaplan, 2006; Kaplan and Rogers, 2013). They also show a preference to view a taxidermic specimen of a wedgetailed eagle with their left eye, and do so just before they move away, presumably in fear (Koboroff et al., 2008).

One might ask what advantage a species gains by specializing the left eye and right hemisphere to detect and respond to predators and, as also found, to specialise the right eye and left hemisphere to detect and pursue food. The answer to this question about the advantage of being lateralized provides evidence against Beauchamp's (2017) speculation that "to perform two activities at the same time (predator detection and food searching) might lead to suboptimal detection or food processing" (pages 4 and 5). The potential advantage of these lateralized specialisations was addressed by an experiment in which chicks were tested in a dual task, searching for grain scattered amongst inedible pebbles and, at the same time, detecting a model predator presented overhead (Rogers et al., 2004). Chicks with stronger lateralization of these particular modes of processing performed better on both aspects of the task than did nonlateralized chicks. Those with stronger lateralization not only detected the predator sooner than did the non-lateralized ones but, after showing initial fear behaviour, they returned to food searching sooner than the non-lateralized chicks. The strongly lateralized chicks also learnt to avoid pecking at the pebbles and to remember this the next day, whereas non-lateralized chicks were unable to learn to avoid pecking at the pebbles and had no memory of the task on the next day. A similar result has been found in strongly versus weakly lateralised fish (Dadda and Bisazza, 2006). In summary, vigilance for predators does not have to compete with search for food, as Beauchamp (2017) and others have suggested. Being able to perform food search simultaneously with predator detection would have obvious value for survival.

As these experiments show, recognition of asymmetry of both vigilance and control of fear responses may lead to stronger correlations between vigilance and fear than found when no allowance is made for side biases. 


\section{References}

Austin, N.P. and Rogers, L.J. (2007) Asymmetry of flight and escape turning responses in horses. Laterality 12: 464-474.

Austin, N.A. and Rogers, L.J. (2012) Limb preferences and lateralization of aggression, reactivity and vigilance in feral horses (Equus caballus). Animal Behaviour 83: 239-247.

Austin, N.A. and Rogers, L.J. (2014) Lateralization of agonistic and vigilance responses in Przewalski horses (Equus przewalskii). Applied Animal Behaviour Science 151: 43-50.

Beauchamp, G. (2017) What can vigilance tell us about fear? Animal Sentience 15(1).

Dadda, M. and Bisazza, A. (2006) Does brain asymmetry allow efficient performance of simultaneous tasks? Animal Behaviour 72: 523-529.

Kaplan, G. and Rogers, L.J. (2013) Stability of referential signaling across time and locations: testing alarm calls of Australian magpies (Gymnorhina tibicen) in urban and rural Australia and Fiji. PeerJ 1: e112; DOI 10.7717/peerj.112.

Koboroff, A., Kaplan, G. and Rogers, L.J. (2008) Hemispheric specialization in Australian magpies (Gymnorhina tibicen) shown as eye preferences during response to a predator. Brain Research Bulletin 76: 304-306.

Lippolis, G., Bisazza, A., Rogers, J. and Vallortigara, G. (2002) Lateralization of predator avoidance responses in three species of toads. Laterality 7: 163-183.

Lippolis, G., Westman, W., McAllan, B.M. and Rogers, L.J. (2005) Lateralization of escape responses in the striped-faced dunnart, Sminthopsis macroura (Dasyuridae: Marsupalia). Laterality 10: 457-470.

MacNeilage, P.F., Rogers, L.J. and Vallortigara, G. (2009) Origins of the left and right brain. Scientific American 301: 60-67.

Morris, J.S., Ohman, A. and Dolan, R.J. (1999) A subcortical pathway to the right amygdala mediating "unseen" fear. Proceedings of the National Academy of Sciences of the U.S.A. 96: 1680-1685.

Rogers, L.J. (2000) Evolution of hemispheric specialisation: Advantages and disadvantages. Brain and Language 73: 236-253.

Rogers, L.J. and Kaplan, G. (2006) An eye for a predator: Lateralization in birds, with particular reference to the Australian magpie. In Behavioral and Morphological Asymmetries in Vertebrates, edited by Y. Malashichev \& W. Deckel. Landes Bioscience: 47-57.

Rogers, L.J., Vallortigara, G. and Andrew, R.J. (2013) Divided brains: The biology and behaviour of brain asymmetries. Cambridge: Cambridge University Press.

Rogers, L.J., Zucca, P. and Vallortigara, G. (2004) Advantage of having a lateralized brain. Proceedings of the Royal Society of London B 271: S420-S422.

Scicli, A.P., Petrovich, G.D., Swanson, L.W. and Thompson, R.F. (2004) Contextual fear conditioning is associated with lateralized expression of the immediate early gene c-fos in the central and basolateral amygdalar nuclei. Behavioral Neuroscience 118: 5-14.

Young, E.J. and Williams, C.L. (2013) Differential activation of amygdala Arc expression by positive and negatively valenced emotional learning conditions. Frontiers in Behavioral Neuroscience Vol. 7: article 191. 